\title{
Review: The endless nutritional and pharmaceutical benefits of the Himalayan
gold, Cordyceps; Current knowledge and prospective potentials
}

\author{
W.A. ELKHATEEB ${ }^{\prime}$, G.M. DABA \\ Pharmaceutical Industries Division, Department of Chemistry of Natural and Microbial Products, National Research Centre. Tahrir Street, 12311, Dokki, \\ Giza, Egypt. `Email: waillahmed@yahoo.com.
}

Manuscript received: 2 July 2020. Revision accepted: 20 July 2020.

\begin{abstract}
Elkhateeb WA, Daba GM. 2020. Review: The endless nutritional and pharmaceutical benefits of the Himalayan gold, Cordyceps; Current knowledge and prospective potentials. Biofarmasi J Nat Prod Biochem 18: 74-81. As a traditional medicine, Cordyceps has long been used in Asian nations for maintaining vivacity and boosting immunity. Numerous publications on various bioactivities of Cordyceps have been investigated in both in-vitro as well as in vivo studies. Nevertheless, the role of Cordyceps is still arguable whether it acts as a food supplement for health benefits or a real healing drug that can be prescribed in medicine. The Cordyceps industry has developed greatly and offers thousands of products commonly available in a global marketplace. This review will focus on introducing the ecology of Cordyceps and their classification. Moreover, elucidation of the richness of extracts originating from this mushroom in nutritional components was presented, with a description of the chemical compounds of Cordyceps and its wellknown compounds such as cordycepin and cordycepic acid. Furthermore, highlights on natural growth and artificial cultivation of famous Cordyceps species were presented. The health benefits and reported bioactivities of Cordyceps species as promising antimicrobial, anticancer, hypocholesterolemic, antioxidant, antiviral, anti-inflammatory, organ protective agent, and enhancer for organ function were presented.
\end{abstract}

Keywords: Cordyceps; cultivation; secondary metabolites; traditional medicine

\section{INTRODUCTION}

Commonly seen nowadays on shelves of pharmacies and drug stores and recommended for many benefits such as boosting immunity, the genus Cordyceps is an ascomycetous traditional medicinal mushroom that is famous for having numerous bioactive compounds. Cordyceps have different common names: insect mushroom, caterpillar fungus, Himalayan gold, etc. Still, the name Cordyceps originates from the Latin words (cord), which means 'club,' and (ceps), referring to 'head.' The fruiting bodies of these fungi appear from the head of different life stages of various orders of insect species (Zhou et al. 2009; Smiderle et al. 2014; Chen et al. 2000; Peterson 2008; Wang and Yao 2011; Dworecka-Kaszak 2014). The genus Cordyceps is classified under the order Hypocreales, family Ophiocordycipitaceae, and phylum Ascomycota. Some genera belong to families Cordycipitaceae and partial Clavicipitaceae, as shown in Table 1 (Pu and Li 1996; Buenz et al. 2005; Sung et al. 2007; Kepler et al. 2012).

Species belonging to the genus Cordyceps have a golden history due to their safe use in traditional oriental medicines (Paterson 2008). Also, they were utilized 2000 years ago in China for curing different infectious diseases (Singh et al. 2008; Zhou et al. 2009). Although Cordyceps has a wide-reaching distribution, most species have been described from Asia (Boesi Alessandro and Francesca Cardi 2009).
The most famous and widely used species of Cordyceps is C. sinensis (Berk.) Sacc (syn. Ophiocordyceps sinensis (Berk.) G.H. Sung, J.M. Sung, Hywel-Jones \& Spatafora). Currently, the preferred scientific name is Cordyceps sinensis (Berk.) Sacc. (Devkota et al. 2006). This species has a wide host range, including different species of Lepidopteran larvae (Wang and Yao 2011), numerous Thitarodes caterpillars, and its most common host, the Himalayan bat moth Hepialus armoricanus (Chen et al. 2000). Cordyceps militaris (also known as orange caterpillar mushroom) is rich in bioactive compounds and hence has medical-biological activities in a similar way to Cordyceps sinensis (Shrestha and Sung 2005; Gong et al. 2006; Ma et al. 2007; Huang et al. 2009; Das et al. 2010; Dong et al. 2012). This review discussed ecology, classification, nutritional components, chemical composition, natural growth and artificial cultivation, health benefits, and reported bioactivities of famous Cordyceps species.

\section{CORDYCEPS ECOLOGY}

Many Cordyceps species grow by feeding on insect larvae and sometimes on mature insects. Cordyceps grows on insects, crickets, cockroaches, bees, centipedes, black beetles, and ants. From the genus Cordyceps, there are several species known to have medical value, only a few are cultivated, and the most common and well-known are 
Cordyceps sinensis (Figure 1) and Cordyceps militaris (Figure 2) (Halpern Georges 2007). Cordyceps may also grow on other arthropods and the fungus Elaphomyces Nees.

Table 1. The current classification system of Cordyceps (Cordyceps sensu lato)

\begin{tabular}{|c|c|}
\hline Genus & No. of species \\
\hline \multicolumn{2}{|c|}{ Partial Clavicipitaceae } \\
\hline Drechmeria & 2 \\
\hline Hypocrella & 50 \\
\hline Metacordyceps & 4 \\
\hline Metarhizium & 35 \\
\hline Nomuraea & 3 \\
\hline Pochonia & 3 \\
\hline Podocrella & 4 \\
\hline Regiocrella & 2 \\
\hline Sphaerocordyceps & 2 \\
\hline Tyrannicordyceps & 5 \\
\hline Total: 10 & 110 \\
\hline \multicolumn{2}{|c|}{ Ophiocordycipitaceae } \\
\hline Blistum & 1 \\
\hline Didymobotryopsis & 3 \\
\hline Elaphocordyceps & 1 \\
\hline Haptocillium & 8 \\
\hline Hirsutella & 78 \\
\hline Hymenostilbe & 22 \\
\hline Ophiocordyceps & 155 \\
\hline Paraisaria & 2 \\
\hline Perennicordyceps & 4 \\
\hline Polycephalomyces & 12 \\
\hline Purpureocillium & 3 \\
\hline Syngliocladium & 5 \\
\hline Synnematium & 1 \\
\hline Tolypocladium & 39 \\
\hline Trichosterigma & 1 \\
\hline Total: 15 & 335 \\
\hline \multicolumn{2}{|l|}{ Cordycipitaceae } \\
\hline Akanthomyces & 13 \\
\hline Ascopolyporus & 7 \\
\hline Beauveria & 31 \\
\hline Beejasamuha & 1 \\
\hline Cordyceps & 175 \\
\hline Coremiopsis & 2 \\
\hline Engyodontium & 5 \\
\hline Gibellula & 21 \\
\hline Hyperdermium & 2 \\
\hline Insecticola & 2 \\
\hline Isaria & 83 \\
\hline Lecanicillium & 21 \\
\hline Microhilum & 1 \\
\hline Phytocordyceps & 1 \\
\hline Pseudogibellula & 1 \\
\hline Rotiferophthora & 27 \\
\hline Simplicillium & 8 \\
\hline Torrubiella & 66 \\
\hline Total: 18 & 467 \\
\hline
\end{tabular}

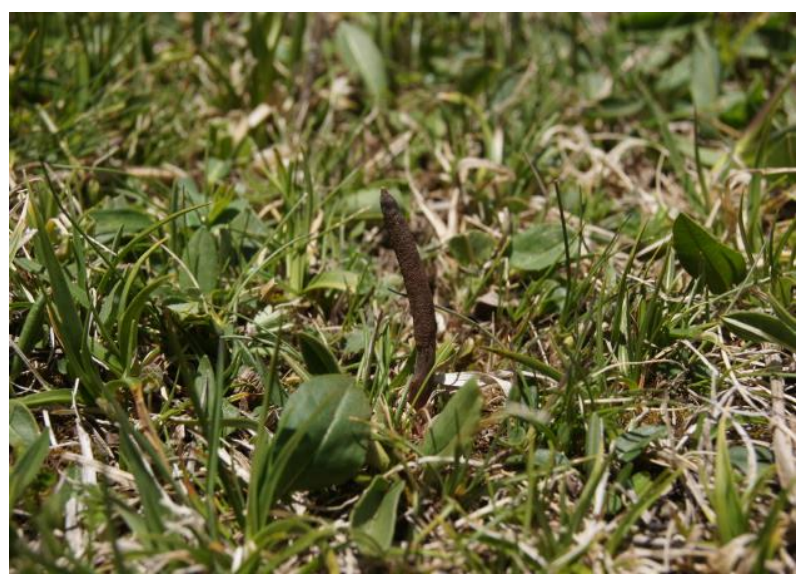

Figure 1. Cordyceps sinensis (Collector: Soraj25: Locality: Nepal, Mid-Western, Jumla, Nepal, hosted by http://mycoportal.org)

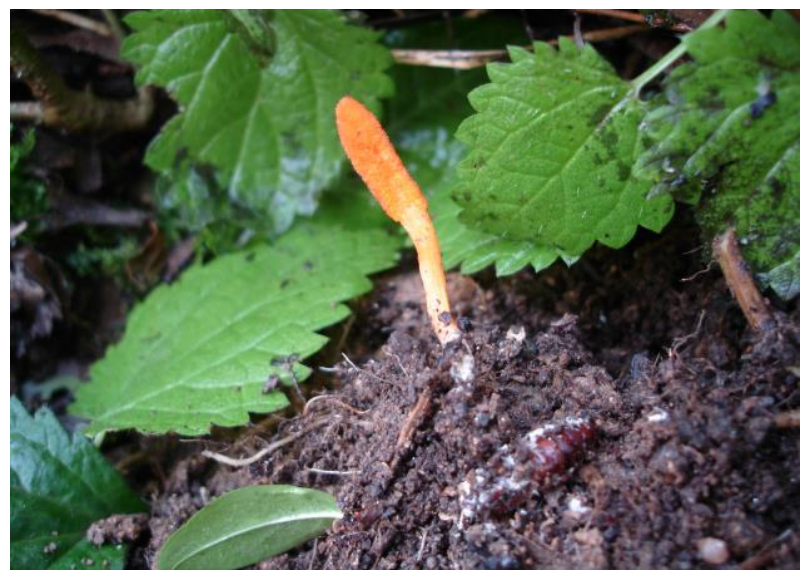

Figure 2. Cordyceps militaris (Collector: Marjan Kustera: Locality: Gabrovac, Serbia, hosted by http://mycoportal.org).

Classification of the genus Cordyceps was previously within the family Clavicipitaceae, order Hypocreales (the old genus Cordyceps Fr.). Currently, many genera have segregated from the genus Cordyceps, such as Metacordyceps, Tyrannycordyceps (placed in family Clavicipitaceae); Elphcordyceps, Ophiocordyceps (in family Ophiocordycepitaceae); Sphaerocordyceps, and remaining Cordyceps species (placed in Incertae sedis within Hypocreales). Due to their special nutritional and healing values, Cordyceps is widely spreading in China, with a huge existing domestic market. In the Chinese market, Cordyceps is known as 'Dongchong Xiacao (worm in winter, an herb in summer; O. sinensis (Berk.) Sung et al. 2007), which is the most expensive type and only produced from the Tibetan Plateau; other Cordyceps species in the markets are termed 'fake Dongchong Xiacao.’ 


\section{THE NUTRITIONAL COMPONENTS IN CORDYCEPS}

Cordyceps is rich in various compounds considered nutritional such as vitamins $(\mathrm{K}, \mathrm{B} 1, \mathrm{~B} 2, \mathrm{~B} 12$, and $\mathrm{E})$; essential amino acids. Additionally, many mono-, di-, and oligosaccharides and many complex polysaccharides were found in Cordyceps besides trace elements (Na, K, Zn, Ca, $\mathrm{Mg}, \mathrm{Al}, \mathrm{Fe}, \mathrm{Cr}, \mathrm{Cu}, \mathrm{Mn}, \mathrm{Zr}, \mathrm{Pi}, \mathrm{Se}, \mathrm{Si}, \mathrm{Sr}, \mathrm{Ti}, \mathrm{Ga}, \mathrm{V}$, and $\mathrm{Ni}$ ), proteins, nucleosides, and sterols. Cordyceps contains considerable quantities of polysaccharides, representing 3$8 \%$ of the mushroom's total weight, and these polysaccharides usually originate from fruiting bodies. Cordyceps polysaccharides are considered the major biologically active compounds besides nucleotides (Zhou et al., 2009; Mishra and Yogesh, 2011; Elkhateeb et al., 2019).

\section{CHEMICAL COMPOSITION OF THE MOST COMMON CORDYCEPS SPECIES}

Cordyceps sinensis (Berk.) Sacc.) is considered the most expensive and well-studied Cordyceps species. According to different chemical analyses, $C$. sinensis contains proteins, polysaccharides, fats, fiber, and carbohydrates, the famous bioactive compounds cordycepin (30-deoxyadenosine) and cordycepic acid (Dmannitol), and different vitamins (Ohta et al. 2007; Zhou et al. 2009).

The therapeutic potentials of Cordyceps depend mainly on the key actions of increased oxygen utilization, ATP production, and sugar metabolism stabilization. Many bioactive compounds originating from Cordyceps are responsible for those effects, such as cordycepin, cordycepic acid, polysaccharides, vitamins, and trace elements. The full bioactive compounds existing in $C$. sinensis are not yet identified. However, at least two compounds, cordycepic acid and cordycepin, have been identified and recommended as important bioactive compounds. Of all Cordyceps species, C. militaris is the only species that has been successfully cultivated and most intensively studied. The majority of Cordyceps products available in the markets are developed from the fruiting bodies of cultivated $C$. militaris. According to reported chemical investigations, C. militaris contains cordycepin, adenosine, polysaccharide, mannitol, trehalose, polyunsaturated fatty acids, $\delta$-tocopherol, $\mathrm{p}$ Hydroxybenzoic acid, and $\beta-(1 \rightarrow 3)-D-g l u c a n$ (Reis et al. 2013; Liu et al. 2014; Smiderle et al. 2014; Wen et al. 2017; Elkhateeb et al. 2019).

\section{CORDYCEPS NATURAL GROWTH AND ARTIFICIAL CULTIVATION}

The natural growing fruiting bodies of Cordyceps are rare, and their collection is an expensive process. Moreover, natural populations of key Cordyceps species are decreasing rapidly due to over-collection (Stone 2008;
Zhang et al. 2012), presenting the need for increased invitro cultivation using the artificial medium. While over 400 species under the genus Cordyceps have been identified, only 36 species have been successfully cultivated in artificial media (Sung et al. 1999; Yin and Qin 2009). The first large scale fruiting techniques used for growing Cordyceps reduced the natural growing cycle from 5 to 2 years; this technique included breeding the host larvae, Thitarodes (Hepialus), then placing about 100 larvae into shoe carton sized plastic containers covered with lids, which are filled with grassland soil containing roots and tubers of their natural foods collected from the wild, besides other roots from cultivation. Spores of $C$. sinensis were inoculated after two years, and about $10 \%$ of the larvae were actually taken over by Cordyceps and grew stromata (Yue et al., 2013). On the other hand, Arora et al. (2013) succeeded in using submerged conditions for culturing Cordyceps sinensis at pH 6 and a temperature of $15^{\circ} \mathrm{C}$.

In previous studies, the medium used for the growth of C. sinensis was sabouraud's dextrose supplemented with yeast extract broth medium, and different additives, carbon, and nitrogen sources were also investigated (Arora et al. 2009). The highest number of conidia was obtained under the physical stress of freeze-shock (Ren and Yao 2013). Sucrose has been reported as the best-tested carbon source for the growth of $C$. Sinensis. Similarly, beef extract and yeast extract were the best nitrogen sources (Seema et al., 2012). Furthermore, using folic acid meaningfully increased the yield, and adding calcium chloride and zinc chloride as micro and macronutrient increased the yield significantly. One of the optimum artificial techniques for culturing $C$. sinensis was utilizing sterile rice media at 9$13^{\circ} \mathrm{C}$ for $40-60$ days. Then to induce stroma production, the temperature was lowered to $4^{\circ} \mathrm{C}$ and $13^{\circ} \mathrm{C}$ for 40 days to develop fruiting bodies (Cao et al. 2015). It should be highlighted that the growth of Cordyceps mycelium is mainly affected by some environmental factors and many factors such as temperature, growth media, and pH (Calam 1971). Still, after testing various media, potato dextrose agar was confirmed to be the optimum medium at $\mathrm{pH}$ ranging between $8.5-9.5$ at $20-25^{\circ} \mathrm{C}$ (Ruhul et al. 2008).

Artificial cultivation is achieved by inoculating reared larvae with cultured strains, and the infected larvae are monitored and fed indoors for one or two years. After that, C. sinensis could be collected. (Lo et al. 2013). C. militaris cultivation is much easier than $C$. sinensis in both solid and liquid media using different carbon and nitrogen sources since $C$. militaris can complete its life cycle when cultured in-vitro (Shrestha et al. 2004, Xiong et al. 2010). The artificial cultivation of $C$. militaris mycelium on synthetic media has recently been advanced, especially for cordycepin production, using different methods such as surface culture (Masuda et al. 2007) and submerged culture (Mao et al. 2005). Usually, C. militaris stromata production requires 35-70 days (Zhang and Liu 1997; Du et al. 2010). Culture duration depends on many conditions, such as the amount of medium, shape, and volume of the culture container. The development of $C$. militaris stroma cultivation in-vitro started with using insects to grow 
stromata by Leatherdale (1970), followed by laboratory trials using various organic substrates by Yue et al. (1982). For commercial production of $C$. militaris stromata, cereals, including rice, have been widely used (Wen et al., 2008; Chen et al., 2011). Also, using substrates such as wheat grains, cottonseed coats, corn cobs, corn grain, bean powder, millet, and sorghum has shown promising results (Chen and Wu 1990; Zhang and Liu 1997; Gao and Wang 2008, Wei and Huang 2009). The optimum organic substrate currently used is a mixture of rice and silkworm pupae (Shrestha et al. 2005, Sung et al. 2006; Jin et al. 2009). Furthermore, brown rice, malt, and soybean were important sources of nutrition for C. militaris compared to chemical media (Xie et al. 2009). C. militaris cultivation needs a relatively low level of nitrogen (Gao et al. 2000) which may explain lower yields when using insects compared to higher yields reached when cereals were used in the culture. Plant hormones such as colchicines, 2, 4-D, and citric acid triamine can promote $C$. militaris stroma production. Also, potassium, calcium, and magnesium salts can increase the yield of fruiting bodies (Xiao et al., 2010).

Mycelia production for the large-scale production of bioactive compounds is also achievable and has been performed in submerged culture (Huang et al., 2006; Xie et al., 2009; Das et al., 2010). C. militaris cultivation has been improved, successfully producing a high yield of stromata and elevated cordycepin content (Sun et al., 2009; Du et al., 2010). The production of fruiting bodies has been studied for three successive generations (Hong et al. 2010; Xiao et al. 2010; Shrestha et al. 2012; Xiaoli et al. 2014).

\section{CORDYCEPS AND HEALTH BENEFITS}

The genus Cordyceps species are extensively studied due to the uncountable number of medical-biological activities used by their extracted compounds, with various medical and nutritional values. In traditional Chinese medicine, the main use of Cordyceps has been to treat asthma and other bronchial conditions and give energy and sexual power. Recent research now confirms the competence of Cordyceps in many other fields. One of the advances of modern research has been the discovery of cordycepin, which has a potent antibacterial action against the majority of bacterial species that have currently developed resistance to other commonly used antibiotics. Cordyceps showed remarkable activity during treating tuberculosis and human leukemia, as shown in many clinical trials in Asia and elsewhere (Halpern Georges 2007). Cordyceps was shown to improve the maximum amount of oxygen and improve respiratory function. Other components produced by Cordyceps sinensis include the deoxynucleoside 2', 3' deoxyadenosine. Also, Quinic acid derived from cordycepin obtained from Cordyceps exerts antimicrobial and antiviral activities. Many studies have reported the potency of Cordyceps sinensis in healing heart rhythm disturbances such as chronic heart failure and cardiac arrhythmia (Mishra and Yogesh, 2011; Wang et al., 2012).

\section{Anticancer activities of Cordyceps}

Various Cordyceps-originated bioactive compounds have a promising anticancer activity that was previously reported (El-Hagrassi et al., 2020). For example, cordycepin showed antitumor activity against B16 melanoma cells (Yoshikawa 2004 and 2007). In addition, cordycepin inhibited the mammalian target of rapamycin complex 1 in gallbladder cancer cells in-vitro, resulting in loss of cancer cell viability and apoptosis (Wong et al. 2010; Ferreira et al. 2010; Wu et al. 2014). C. militaris was found to inhibit U937 cell growth in a dose-dependent manner and limit human leukemia (Park 2005). Cordyceps has shown promising results in slowing and inhibiting the growth of cancer cells (Santhosh Kumar et al. 2014) and, in some cases, could reduce tumor size (Nakamura et al. 2003). Clinical trials on cancer patients have been conducted in many Asian countries, showing talented results in reducing tumor size (Wang et al. 2001), improving tolerance for chemotherapy and/or radiation (Zhou et al. 1998), and stimulating the immune system which, hence, enhances the efficiency of chemotherapy (Shin et al. 2003). Crude extract of C. militaris showed potent anticancer activity in a xenograft mouse model with RMA cell-derived tumors (Park et al., 2017). Additionally, some Cordyceps species have anti-leukemia activities and better suppressive effects of chemotherapy on bone marrow function as a model for cancer treatment (Liu et al. 2008; Wong et al. 2010; Ferreira et al. 2010).

\section{Hypoglycemic and hypocholesterolemic effects}

Cordyceps are found to regulate and lower blood sugar levels by improving glucose metabolism and conserving hepatic glycogen (Zhao et al., 2002; El-Hagrassi et al., 2020). Cordyceps can increase the secretion of glucokinase and hexokinase, which are glucose-regulating enzymes secreted by the liver (Kim et al., 2017). Polysaccharides are the key player in showing the hypoglycaemic activity of Cordyceps. For example, CS-F30, a polysaccharide extracted from $C$. sinensis culture mycelium, has been reported for its promising hypoglycaemic effect (Kiho 1996). Additionally, the plasma glucose level was reduced quickly after intravenous administration of CS-F30 in normal and streptozocin-induced diabetic mice (Kiho 1996). Another polysaccharide (CS-F10) was purified from a hot-water extract of $C$. sinensis cultured mycelia and consists of galactose, glucose, and mannose in a molar ratio of 43:33:24. CS-F10 lowers the plasma glucose level in normal, adrenaline-induced hyperglycaemic, and diabetic mice. Hypercholesterolemia is an indicator of a high risk of cardiovascular attack. El-Hagrassi et al. (2020) reported the role of $C$. militaris in lowering the total cholesterol level and the level of triglycerides. A hot-water extract of $C$. sinensis mycelia has been found to reduce total cholesterol concentration in the serum of mice by reducing LDL (lowdensity lipoprotein) hypocholesterolemic activities (ElHagrassi et al., 2020). A hot-water extract of $C$. sinensis mycelia has been found to reduce total cholesterol concentration in the serum of mice by reducing LDL) and very-low-density lipoprotein and increasing good cholesterol concentration (HDL cholesterol) (Koh 2003). 


\section{Improving kidney functions and liver disorders}

Some clinical trials revealed that the administration of C. sinensis could significantly improve kidney function and boost the overall immunity of patients diagnosed with chronic renal failure (Guan et al. 1992). Additionally, treating patients with gentamicin-induced kidney damage helped recover $89 \%$ of normal kidney function in a relatively short time (Zhou et al. 1990). The mode of kidney enhancing the action of Cordyceps is explained by its ability to protect sodium pump activity of tubular cells, increase 17- ketosteroid and 17-hydroxycorticosteroid levels in the human body, reduce the content of calcium in certain tissues, and accelerate the regeneration of tubular cells (Zhou et al. 1990; Xu et al. 1995; Wang et al. 1998). Cordyceps is commonly used for the treatment of chronic hepatitis B and C. Using the antiviral drug, lamivudine, plus mixed extracts of Cordyceps with other medicinal mushrooms showed promising results for treating hepatitis B (Wang and Shiao 2000; Ng and Wang, 2005; Zhou et al. 2009). On the other hand, daily consumption of Cordyceps improved liver functions in patients who have post-hepatic cirrhosis (Zhu and Liu 1992; Zhou et al. 2009). Cordyceps extracts are used to help in the healing of both chronic hepatitis B and C (Wang and Shiao 2000).

\section{Cordyceps as antioxidant and antiaging activities}

One of the most potent bioactivities reported for Cordyceps extracts is their ability to protect cells from being damaged by free radicals. This activity corresponds to polysaccharide fraction ( $\mathrm{Yu}$ et al. 2007; Wang et al. 2012). Cordyceps sinensis has potent antioxidant and antiaging properties (Yamaguchi et al., 2000; Ji et al., 2009). Also, many studies elucidated the antioxidant effect of extracts obtained from $C$. militaris (El-Hagrassi et al., 2020). The fruiting bodies extract of $C$. militaris showed strong DPPH radical scavenging activity, which indicated high antioxidant activity. In contrast, the fermented mycelia extract had stronger total antioxidant activity and reduced capability (Dong et al. 2014). Cordyceps has been used for centuries as a remedy for weakness and fatigue by residents living in the high mountains of Tibet to give them energy which is achieved by increasing cellular ATP (Holliday et al. 2008). Nowadays, athletes utilize Cordyceps to overcome weakness and fatigue, increase endurance and boost energy levels (Liu et al. 1997). Moreover, clinical trials connecting chronic fatigue with aging patients revealed that treatment with $C$. sinensis improved fatigue and dizziness, increasing cold intolerance and amnesia (Mizuno et al. 1999; Chen et al. 2013; Wu et al. 2014).

\section{Cordyceps for organs and glands protection}

Cordyceps sinensis also has clear effects on other organ systems (Chen 1995; Zhang and Yuan 1997; Guo and Guo 2000; Xu 2006). For example, C. sinensis has sedative, anticonvulsant, and cooling effects on the central nervous system. In the respiratory system, $C$. sinensis has a potent relaxant action on bronchi, noticeably increases secretion of adrenaline from the adrenal glands, and participates in tracheal contraction caused by histamine; it also has an expectorant antitussive and anti-asthmatic effects, and it also inhibits pulmonary emphysema. In the endocrine system, $C$. sinensis has effects as a male hormone. Polysaccharides extracted from Cordyceps can increase corticosterone levels in plasma. Cordyceps has been used in traditional medicine for decades to improve men's fertility. A study has proven the positive effect of using $C$. militaris mycelium on sperm motility, morphology, productivity, and enhancement of sexual activity (Lin et al. 2007). Cordyceps extracts contain adenosine, deoxyadenosine, corresponding adenosine type nucleotides, and nucleosides, which help stabilize heartbeat and correct heart arrhythmias (Pelleg and Porter 1990).

\section{Cordyceps as anti-inflammatory}

Commonly, the famous cordycepin is the compound causing the anti-inflammatory activity of numerous Cordyceps species (Won and Park 2005; Kim et al. 2006; Yang et al. 2011). C. militaris ethanolic extracts of cultured fruiting bodies and mycelia exerted an anti-inflammatory activity on the chick embryo chorioallantoic membrane angiogenesis and the croton oil-induced ear edema in mice (Won and Park 2005). On the other hand, an alkaline extract of $C$. militaris showed a strong anti-inflammatory effect against formalin-induced nociception and LPSinduced peritonitis in mice due to containing a potent antiinflammatory compound (linear b- (1R3)-D-glucan) (Smiderle et al. 2014; Park et al. 2015). Adenosine is also extracted from Cordyceps species with numerous activities related to avoiding tissue damage as anti-inflammatory properties (Nakav et al. 2008; Tsai et al. 2010; Liu et al. 2015).

\section{Cordyceps as antiviral agents}

Many studies reported that cordycepin extracted from C. militaris could inhibit infection with the human immunodeficiency virus (Mueller et al. 1991). Cordycepin has shown antiviral activities against different viruses such as plant viruses and human viruses (influenza virus, Epstein-Barr virus, murine leukemia virus) (Ryu et al. 2014). The mode of killing or inhibiting actions of cordycepin is not fully understood, but several studies have elucidated the ability of cordycepin to inhibit numerous protein kinases (Glazer and Kuo 1977; Jin et al. 2011; Elkhateeb et al. 2019). On the other hand, crude extract of fruiting bodies of $C$. militaris showed promising in-vitro antirotavirus SA-11 agent activities (El-Hagrassi et al., 2020).

\section{Cordyceps side effects and safety}

Cordyceps is generally safe in the recommended dosage, and no major side effects were reported. (Das et al. 2010).

\section{CONCLUDING REMARK}

Cordyceps and its bioactive components and metabolites are golden mines with therapeutic potential against various fatal diseases. Developing new techniques 
capable of cultivating species other than Cordyceps militaris can contribute to producing enough quantities of bioactive compounds such as cordycepin and cordycepic acid, which also may lower the cost of this expensive medicinal mushroom. On the other hand, further in vivo studies should be conducted in order to evaluate the clinical activities of Cordyceps metabolites which can be a step toward certifying its use as a medical drug.

\section{REFERENCES}

Arora RK, Singh N, Singh RP. 2013. Characterization of an entomophagous medicinal fungus Cordyceps sinensis (Berk.) Sacc. of Uttarakhand, India. Bioscan 8: 195-200.

Arora RK, Singh RP. 2009. Effect of nutritional sources on mycelial growth of Caterpillar mushroom Cordyceps sinensis (Berk.) Sacc. J Mycol Plant Pathol 39: 114-117.

Boesi A, Francesca C. 2009. "Cordyceps sinensis medicinal fungus: traditional use among Tibetan people, harvesting techniques, and modern uses. Herbal Gram 83: 52-61.

Buenz EJ, Bauer BA, Osmundson TW, Motleym TJ. 2005. The traditional Chinese medicine Cordyceps sinensis and its effects on apoptotic homeostasis, J Ethnopharmacol 96(1-2): 19-29.

Calam CT. 1971. The evaluation of mycelial growth. In: Norris J.R. and Ribbons D.W. (eds). Methods in Microbiology. Academic Press, New York.

Cao L, Ye Y, Han RF. 2015. Fruiting body production of the medicinal Chinese caterpillar mushroom Ophiocordyceps sinensis in artificial medium. Int. J. Med. Mushrooms 17: 1107-12.

Chen DG. 1995. Effects of Jinshuibao capsule on the quality of life of patients with heart failure. J Admin Tradit.Chin Med 5: 40-43.

Chen PX, Wang S, Nie S, Marcone M. 2013. Properties of Cordyceps sinensis: A review. J Functional Foods 5 (2): 550-569.

Chen S, Yin D, Li L, Zha X, Shuen J, Zhama C. 2000. Resources and distribution of Cordyceps sinensis in Naqu Tibet. Zhong Yao Cai 23: 673-675.

Chen SZ, Wu PJ. 1990. A brief introduction to bottle culture technique of Cordyceps militaris. Edible Fungi 4: 31.

Chen YS, Liu BL, Chang YN. 2011. Effects of light and heavy metals on Cordyceps militaris fruit body growth in rice grain-based cultivation. Korean J Chem Eng 28: 875-879.

Das SK, Masuda M, Sakurai A. 2010. Medicinal uses of the mushroom Cordyceps militaris: Current state and prospects. Fitoterapia 81: 961 968.

Devkota S Yarsagumba 2006. [Cordyceps sinensis (Berk.) Sacc.] Traditional utilization in Dolpa District, Western Nepal. Our Nature 4: $48-52$.

Dong C, Yang T, Lian T. 2014. A comparative study of the antimicrobial, antioxidant, and cytotoxic activities of methanol extracts from fruit bodies and fermented mycelia of caterpillar medicinal mushroom Cordyceps militaris (Ascomycetes). International $\mathrm{J}$ medicinal mushrooms 16(5).

Dong JZ, Lei C, Ai XR. 2012. Selenium enrichment on Cordyceps militaris Link and analysis on its main active components. Appl Biochem Biotechnol 166: 1215-1224.

Du AL, Zhang X, Zhang HZ. 2010. A new high cordycepin Cordyceps militaris cultivar 'Haizhou 1'. Acta Hortic Sin. 37: 1373-1374.

Dworecka-Kaszak B. 2014. Cordyceps fungi as natural killers, new hopes for medicine and biological control factors. Ann Parasitol 60(3).

El-Hagrassi A, Daba G, Elkhateeb W, Ahmed E, Negm El-Dein A, Fayad W, Shaheen M, Shehata R, El-Manawaty M, Wen TC. 2020. In-vitro bioactive potential and chemical analysis of the n-hexane extract of the medicinal mushroom, Cordyceps militaris. Malaysian J Microbiol 16 (1): 40-48.

Elkhateeb WA, Daba, GM, Thomas PW, Wen TC. 2019. Medicinal mushrooms as a new source of natural therapeutic bioactive compounds. Egyptian Pharmaceutical Journal 18 (2): 88-101.

Ferreira I, Vaz J, Vasconcelos M, Martins A. 2010. Compounds from wild mushrooms with antitumor potential. Anti-Cancer Agents in Medicinal Chemistry (Formerly Current Medicinal Chemistry-AntiCancer Agents) 10 (5): 424-436.
Gao SY, Wang FZ. 2008. Research of commercialized cultivation technology on Cordyceps militaris. North Horti. 9: 212-215.

Gao X, Wu W, Qian G. 2000. Study on influences of abiotic factors on fruit body differentiation of Cordyceps militaris. Acta Agric Shanghai 16: 93-98.

Glazer RI, Kuo JF 1977. Inhibitory effects of cordycepin on cyclic nucleotide-dependent and cyclic nucleotide-independent protein kinases. Biochemical Pharmacol. 26(14): 1287-1290.

Gong CL, Pan ZH, Zheng XJ. 2006. Antioxidation of cultured Cordyceps militaris growing on silkworm pupa. In: Proceedings of International Workshop on Silk handcrafts cottage industries and silk enterprises development in Africa, Europe, Central Asia and the Near East, \& Second Executive Meeting of Black, Caspian seas and Central Asia Silk Association (BACSA), Bursa, Turkey 615-620.

Guan Y, Hu G, Hou M, Jiang H, Wang X, Zhang C. 1992. Effect of Cordyceps sinensis on lymphocyte subsets in chronic renal failure. Chinese J Integrated Medicine 323: 338-339.

Guo TF, Guo TY. 2000. Study on pharmacological action and clinical application of Cordyceps sinensis. J Jindongnan Teachers Coll 21: 7073.

Halpern Georges 2007. Healing mushrooms. Square One Publishers, Inc. 2007.

Holliday J, Cleaver M. 2008. Medicinal value of the caterpillar fungi species of the genus Cordyceps (Fr.) Link (Ascomycetes). A Review Intl J Med Mushrooms 10: 219-234.

Hong IP, Pil-Don K, Ki-Young K, Sung-Hee N, Man-Young L, Yong-Soo C, Kwang-Gill L, Richard A. 2010. Fruit body formation on silkworm by Cordyceps militaris. Mycobiology 38: 128-132.

Huang L, Li QZ, Chen YY. 2009. Determination and analysis of cordycepin and adenosine in the products of Cordyceps spp. African Journal Microbiol Res 3: 957-961.

Huang SJ, Tsai SY, Lee YL. 2006. Non-volatile taste components of fruiting bodies and mycelia of Cordyceps militaris. Food Sci Technol 39: 577-583.

Ji DB, Ye J, Li CL, Wang YH, Zhao J, Cai SQ. 2009. Antiaging effect of Cordyceps sinensis extract. Phytotherapy Research 23 (1): 116-122)

Jin LY, Du ST, Ma L. 2009. Optimization on mathematical model of basic medium of Cordyceps militaris cultivation. J Northwest A F Univ (Nat Sci Ed) 37: 175-179.

Jin ML, Park SY, Kim YH, Park G, Son HJ, Lee SJ. 2011. Suppression of $\alpha$-MSH and IBMX-induced melanogenesis by cordycepin via inhibition of CREB and MITF, and activation of PI3K/Akt and ERKdependent mechanisms. International $\mathrm{J}$ molecular medicine 29 (1): 119-124.

Kepler RM, Sung GH, Harada Y, Tanaka K, Tanaka E, Hosoya T, Bischoff JF, Spatafora JW. 2012. Host jumping onto close relatives and across kingdoms by Tyrannicordyceps (Clavicipitaceae) gen. nov. and Ustilaginoidea (Clavicipitaceae). Am J Bot 99: 1-10.

Kiho T. 1996. Polysaccharides in fungi, hypoglycemic activity of a polysaccharide (CS-F30) from the cultural mycelium of Ophiocordyceps sinensis and its effect on glucose metabolism in mouse liver. Biol Pharm Bull. 19: 294-296.

Kim DJ, Kang YH, Kim KK, Kim TW, Park JB, Choe M. 2017. Increased glucose metabolism and alpha-glucosidase inhibition in Cordyceps militaris water extract-treated HepG2 cells. Nutrition Research and Practice 11 (3): 180-189.

Kim HG, Shrestha B, Lim SY, Yoon DH, Chang WC, Shin D, Han SK, Park SM, Park JH, Park HI, Sung JM, Jang Y, Chung N, Hwang KC, Kim TW. 2006. Cordycepin inhibits lipopolysaccharide-induced inflammation by the suppression of NF- $\mathrm{B}$ through Akt and p38 inhibition in RAW 264.7 macrophage cells. European J Pharmacol 545 (2-3): 192-199.

Koh JH. 2003. Hypocholesterolemic effect of hot water extract from mycelia of Cordyceps sinensis. Biol Pharm Bull 26: 84-87.

Leatherdale D. 1970. The arthropod hosts of entomogenous fungi in Britain. Entomophaga 15: 419-435.

Lin WH, Tsai MT, Chen YS, Hou RCW, Hung HF, Li CH, Jeng KC. 2007. Improvement of sperm production in subfertile boars by Cordyceps militaris supplement. The American J Chinese medicine 35 (04): 631-641.

Liu J, Yang S, Yang X, Chen Z, Li J. 1997. Anticarcinogenic effect and hormonal effect of Cordyceps militaris Link. Chung Kuo Chung Yao Tsa Chih (Chinese Journal); 22: 111-113.

Liu X, Huang K, Zhou J. (2014). Composition and antitumor activity of the mycelia and fruiting bodies of Cordyceps militaris. J Food Nutr Res 2 (2): 74-79. 
Liu Y, Wang J, Wang W, Zhang H, Zhang X, Han C. 2015. The chemical constituents and pharmacological actions of Cordyceps sinensis. Evid-Based Compl Altern Med 2015: 575063. DOI: $10.1155 / 2015 / 575063$.

Lo HC, Hsieh C, Lin FY, Hsu TH. 2013. A Systematic Review of the Mysterious Caterpillar Fungus Ophiocordyceps sinensis in Dong Chong Xia Cao (冬蟲夏草 Dōng Chóng Xià Căo) and related bioactive ingredients. J Trad Compl Med 3 (1): 16-32.

Ma T, Feng Y, Wu XP 2007. Primary investigation of a host insect of Cordyceps militaris and analysis of its main ingredients. For Res 20: 63-67.

Mao XB, Eksriwong T, Chauvatcharin S, Zhong J. 2005. Optimization of carbon source and $\mathrm{C}: \mathrm{N}$ ratio for cordycepin production by submerged cultivation of medicinal mushroom Cordyceps militaris. Process Biochem. 40: 1667-1672.

Masuda M, Urabe E, Honda H, Sakurai A, Sakakibara M. 2007. Enhanced production of cordycepin by surface culture using the medicinal mushroom Cordyceps militaris. Enzyme Microb Tech 40: 1199-1205.

Mishra R, Yogesh U. 2011. Cordyceps sinensis: The Chinese Rasayancurrent research scenario. Intl J Res Pharmaceut Biomed Sci 2 (4): 1503-1519.

Mizuno T. 1999. Medicinal effects and utilization of Cordyceps (Fr.) Link (Ascomycetes) and Isaria Fr. (Mitosporic fungi) Chinese caterpillar fungi, "Tochukaso" (review). Intl J Med Mushrooms 1: 251-262.

Mueller WE, Weiler BE, Charubala R, Pfleiderer W, Leserman L, Sobol RW, Suhadolnik RJ, Schroeder HC. 1991. Cordycepin analogs of 2', 5 '-oligoadenylate inhibit human immunodeficiency virus infection via inhibition of reverse transcriptase. Biochemistry 30: 2027-2033.

Nakamura K, Konoha K, Yamaguchi Y, Kagota S, Kazumasa S, Kunitomo M. 2003. Combined effects of Cordyceps sinensis and methotrexate on hematogenic lung metastasis in mice. Receptors Channels 9: 329-334

Nakav S, Chaimovitz C, Sufaro Y, Lewis EC, Shaked G, Czeiger D, Zlotnik M, Douvdenvani A. 2008. Anti-inflammatory preconditioning by agonists of adenosine A1 receptor. PLoS ONE 3 (5): e2107. DOI: 10.1371/journal.pone.0002107.

$\mathrm{Ng}$ TB, Wang HX. 2005. Pharmacological actions of Cordyceps, a prized folk medicine. J Pharm Pharmacol 57 (12): 1509-1519.

Ohta Y, Lee JB, Hayashi K, Fujita A, Park DK, Hayashi T. 2007. In vivo anti-influenza virus activity of an immunomodulatory acidic polysaccharide isolated from Cordyceps militaris grown on germinated soybeans. J Agric Food Chem 55 (25): 10194-10199.

Park C. 2005. Growth inhibition of U937 leukemia cells by aqueous extract of Cordyceps militaris through induction of apoptosis. Oncol Rep 13: 1211-1216.

Park SY, Jung SJ, Ha KC, Sin HS, Jang SH, Chae HJ, Chae SW. 2015. Anti-inflammatory effects of Cordyceps mycelium (Paecilomyces hepiali, CBG-CS-2) in Raw264. 7 murine macrophages. Oriental Pharma Exp Med 15 (1): 7-12.

Park J, Lee Y, Baek, T, Yoon, N, Kim D, Aravinthan T, Hong A, HoonKim GJ. 2017. Anticancer efficacy of Cordyceps militari. ethanol extract in a xenografted leukemia model. Evid-Based Compl Altern Med 2017: 8474703. DOI:

Paterson RR. 2008. Cordyceps-A traditional Chinese medicine and another fungal therapeutic biofactory? Phytochemistry 69 (7): 1469 1495.

Pelleg A, Porter R. 1990. The pharmacology of adenosine. Pharmacotherapy (10): 157-174.

Pu ZL, Li ZZ. 1996. Insect Mycology. Anhui Science and Technology Press, Hefei, 108.

Reis FS, Barros L, Calhelha RC, Ćirić A, Van Griensven LJ, Soković M, Ferreira IC. 2013. The methanolic extract of Cordyceps militaris (L.) Link fruiting body shows antioxidant, antibacterial, antifungal and antihuman tumor cell lines properties. Food Chem Toxicol 62: 91-98.

Ren SY, Yao YJ. 2013. Evaluation of nutritional and physical stress conditions during vegetative growth on conidial production and germination in Ophiocordyceps sinensis. FEMS Microbiol Lett 346 (1): 29-35.

Ruhul AS, Nadia A, Mousumi T, Asaduzzaman KM. 2008. Study of mycelial growth of Cordyceps sinensis in different media, at different $\mathrm{pH}$ level and temperature. Bangladesh J Mushroom 2: 43-48.

Ryu E, Son M, Lee M, Lee K, Cho J.Y, Cho S, Lee S.K, Lee Y.M, Cho H, Sung GH, Kang H. 2014. Cordycepin is a novel chemical suppressor of Epstein-Barr virus replication. Oncoscience 1 (12): 866.
Santhosh KT, Sujathan K, Biba V. 2014. Naturally occurring entomogenous fungi having anti-cancerous properties. Proceedings of the 25th Swadeshi Science Congress Kerala 206: 97.

Seema S, Subir R, Prem SN, Mohammed A. 2012. Optimization of nutritional necessities for in-vitro culture of Ophiocordyceps sinensis. Intl J Sci. Res 3: 1523-1528.

Shin KH, Lim SS, Lee S, Lee YS, Jung SH, Cho SY. 2003. Anti-tumour and immunostimulating activities of the fruiting bodies of Paecilomyces japonica, a new type of Cordyceps spp. Phytother Res 17: 830-833.

Shrestha B, Han SK, Lee WH. 2005. Distribution and in-vitro fruiting of Cordyceps militaris in Korea. Mycobiology 33: 178-181.

Shrestha B, Park YJ, Han SK. 2004. Instability in in-vitro fruiting of Cordyceps militaris. J Mushroom Sci Prod 2: 140-144.

Shrestha B, Sang KH, Sung JM, Sung GH. 2012. Fruiting body formation of Cordyceps militaris from multi-ascospore isolates and their single ascospore progeny strains. Mycobiology 40: 100-106.

Shrestha B, Sung J. 2005. Notes on Cordyceps species collected from central region of Nepal. Mycobiology 33: 235-239.

Singh RP, Pachauri V, Verma RC, Mishra KK. 2008. Caterpillar fungus (Cordyceps sinensis) - A Review. J Ecofriendly Agric 3: 1-15.

Smiderle FR, Baggio CH, Borato DG, Santana-Filho AP, Sassaki GL, Iacomini M, Van Griensven LJ. 2014. Anti-inflammatory properties of the medicinal mushroom Cordyceps militaris might be related to its linear $(1 \rightarrow 3)-\beta$-D-glucan. PloS One 9 (10): e110266. DOI: 10.1371/journal.pone.0110266.

Stone R. 2008. Last stand for the body snatcher of the Himalayas? Science 322: 1182.

Sun JD, Xiong ST; Wang P. 2009. Study on biological and cultivated characters of Cordyceps militaris SN3. J Fungal Res 7 (324): 148152.

Sung GH, Hywel-Jones NL, Sung JM, Luangsa-ard JJ, Shrestha B, Spatafora JW. 2007. Phylogenetic classification of Cordyceps and the clavicipitaceous fungi. Stud Mycol. (57): 5-69.

Sung JM, Choi YS, Lee HK. 1999. Production of fruiting body using cultures of entomopathogenic fungal species. Korean J. Mycol. 27: $15-19$.

Sung JM, Park YJ, Lee JO. 2006. Effect of preservation periods and subcultures on fruiting body formation of Cordyceps militaris invitro. Mycobiology (34): 196-199.

Tsai YJ, Lin LC; Tsai TH. 2010. Pharmacokinetics of adenosine and cordycepin, a bioactive constituent of Cordyceps sinensis in rat. J Agric Food Chem 58 (8): 4638-4643.

Wang M, Meng XY, Le Yang R, Qin T, Wang XY, Zhang KY, Fei C, Li Y, Hu Y, Xue F. 2012. Cordyceps militaris polysaccharides can enhance the immunity and antioxidation activity in immunosuppressed mice. Carbohydrate Polymers 89 (2): 461-466.

Wang R, Xu Y, Ji P, Wang X, Holliday J. 2001. Clinical trial of a mixture of six medicinal mushroom extracts. http://wwwalohamedicinals.com/c linical_trials.html

Wang SM, Lee LJ, Lin WW, Chang CM. 1998. Effects of a water-soluble extract of Cordyceps sinensis on steroidogenesis and capsular morphology of lipid droplets in cultured rat adrenocortical cells. J Cell Biochem 69: 483-489.

Wang SY. Shiao MS. 2000. Pharmacological functions of Chinese medicinal fungus Cordyceps sinensis and related species. J Food Drug Anal 8: 84-88.

Wang XL, Yao YJ. 2011. Host insect species of Ophiocordyceps sinensis: A review. Zookeys 127: 43-59.

Wei Q, Huang M. 2009. Effects of nutrient ingredient in culture medium on the growth of Cordyceps militaris. Beijing Agric 27: 36-38.

Wen TC, Kang JC, Li GR. 2008. Effects of different solid culture condition on fruit body and cordycepin output of Cordyceps militaris. Guizhou Agric Sci 36: 92- 94

Wen TC, Zha LS, Kang JC, Hyde KD. 2017. Problems and prospects of research and development of Cordyceps militaris. Mycosystema 36: 14-27.

Won SY, Park EH. 2005. Anti-inflammatory and related pharmacological activities of cultured mycelia and fruiting bodies of Cordyceps militaris. J Ethnopharmacol 96 (3): 555-561.

Wong YY, Moon A, Duffin R, Barthet-Barateig A, Meijer HA, Clemens MJ, De Moor CH. 2010. Cordycepin inhibits protein synthesis and cell adhesion through effects on signal transduction. J Biol Chem. 285: 2610-2621.

Wu JY, Leung HP, Wang WQ, Xu C. 2014. Mycelial fermentation characteristics and anti-fatigue activities of a Chinese caterpillar 
fungus, Ophiocordyceps sinensis strain Cs-HK1 (Ascomycetes). Intl J Med Mushrooms 16 (2): 105-114.

Xiao ZH, Li ZX, Li JZ. 2010. Influence of additive on growth and differentiation of Cordyceps militaris (L.) fruit body. Food Ferment Technol 46: 60-64.

Xiaoli L, Kaihong H, Jianzhong Z. 2014. Composition and antitumor activity of the mycelia and fruiting bodies of Cordyceps militaris." J Food Nutrition Research (2): 74-79.

Xie CY, Gu ZX, Fan GJ. 2009. Production of cordycepin and mycelia by submerged fermentation of Cordyceps militaris in mixture natural culture. Appl Biochem Biotechnol (158): 483-492.

Xiong CH, Xia YL, Zheng P. 2010. Developmental stage-specific gene expression profiling for a medicinal fungus Cordyceps militaris. Mycology 1: 25- 66.

Xu CD. 2006. Advances of researches on Cordyceps sinensis. J Fungal Res 4: 60-64.

Xu F, Huang JB, Jiang L, Xu J, Mi J. 1995. Amelioration of cyclosporin nephrotoxicity by Cordyceps sinensis in kidney-transplanted recipients. Nephrology Dialysis Transplantation 10 (1): 142-143.

Yamaguchi Y, Kagota S, Nakamura K, Shinozuka K, Kunitomo M. 2000 Antioxidant activity of the extracts from fruiting bodies of cultured Cordyceps sinensis. Phytotherapy Research: An International Journal Devoted to Pharmacological and Toxicological Evaluation of Natural Product Derivatives 14 (8): 647-649.

Yang ML, Kuo PC, Hwang TL, Wu TS. 2011. Anti-inflammatory principles from Cordyceps sinensis. J Nat Prod 74 (9): 1996-2000.

Yin H, Qin S. 2009. Effects of cultivation conditions on cell growth of inoculums of Cordyceps sinensis. Modern Food Sci Technol 25 (2): 188-190.

Yoshikawa N. 2004. Antitumor activity of Cordycepin in mice. Clin Exp Pharmacol Physiol 31: S51-S53.

Yoshikawa N. 2007. Cordycepin and Cordyceps sinensis reduce the growth of human promyelocytic leukaemia cells through the Wnt signaling pathway. Clin Exp Pharmacol Physiol 34: S61-S63.
Yu R, Yang W, Song L, Yan C, Zhang Z, Zhao Y. 2007. Structural characterization and antioxidant activity of a polysaccharide from the fruiting bodies of cultured Cordyceps militaris. Carbohydr Polym (70): 430-436.

Yue DC, Yang YP, Wang SF. 1982. Preliminary study on stroma formation of Cordyceps militaris strain. J Chin Med Mater (5): 7-10.

Yue K, Ye M, Zhou Z. 2013. The artificial cultivation of medicinal caterpillar fungus, Ophiocordyceps sinensis (Ascomycetes): A review. Intl J Med Mushrooms 15: 425-434.

Zhang CK, Yuan SR. 1997. Recent advances in studies on immune pharmacological activities of Cordyceps sinensis and its submergecultured mycelia. J Capital University Med Sci (18): 37-38.

Zhang XK, Liu WX. 1997. Experimental studies on planting Cordyceps militaris (L. ex Fr.) Link with different culture materials. Edible Fungi China 16: 21-22.

Zhang YJ, Li E, Wang CS. 2012. Ophiocordyceps sinensis, the flagship fungus of China: Terminology, life strategy and ecology. Mycology 3: 2-10.

Zhao CS, Yin WT, Wang JY, Zhang Y, Yu H, Cooper R. 2002. Cordyceps Cs-4 improves glucose metabolism and increases insulin sensitivity in normal rats. J Altern Compl Med 8: 403-405.

Zhou JS, Halpern G, Jones K. 1998. The scientific rediscovery of an ancient Chinese Herbal Medicine: Cordyceps sinensis. J Alternat Compl Med 4: 429-457.

Zhou L. 1990. Short term curative effect of cultured Cordyceps sinensis (Berk) Sacc. Mycelia in Chronic Hepatitis B. Chang Kuo Chung Yao Tsa Chih (Chinese Journal) 19: 53-55. [Chinese]

Zhou X, Gong Z, Su Y, Lin J, Tang K. 2009. Cordyceps fungi: natural products, pharmacological functions and developmental products. J Pharm Pharmacol 61 (3): 279-291.

Zhu J, Liu C. 1992. "Modulating effects of extractum semen Persicae and cultivated Cordyceps hyphae on immuno-dysfunction of inpatients with posthepatitic cirrhosis. J Integr Trad Western Med 12 (4): 207209. [Chinese] 